\title{
Wonderful experience in and outside the Ampara hospital
}

\author{
Bower G, Lee M, Cooper D, Croft H
}

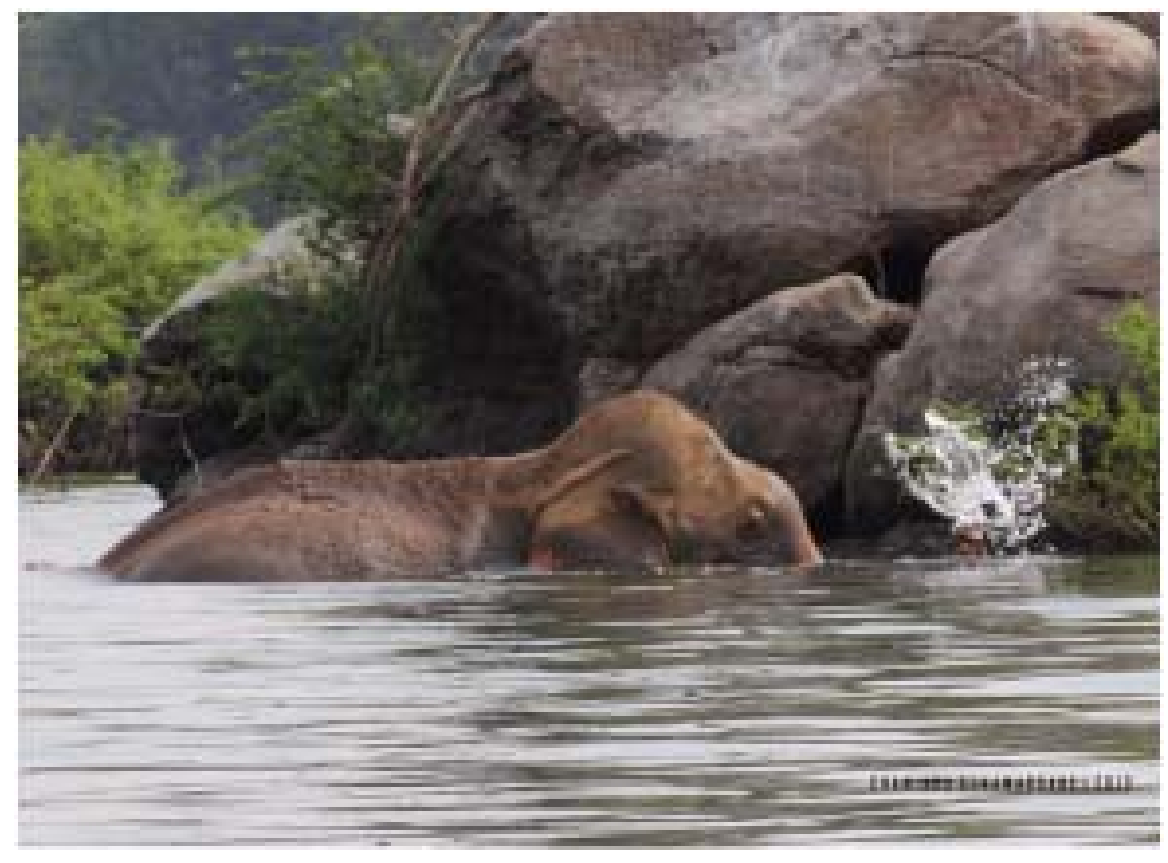

As final year medical students from St George's, University of London, we were required to do a medical elective after our final exams: a placement in a healthcare setting anywhere in the world in any specialty that interested us. We chose Sri Lanka for a number of reasons. St George's Hospital has a great deal of Sri Lankan doctors working in it, and there is a large Sri Lankan community in the surrounding area. From our public health studies, we knew that Sri Lanka has an enviable health care system in which patients have good access to a large range of health services for free. Markers of good health such as maternal and infant mortality and life expectancy are all high when compared to other developing nations.

St George's University of London.

Correspondence: Guy Bower

E-mail: guybower@ hotmail.co.uk
Given the spending difference between Sri Lanka and the UK, we were interested to see how this has been achieved, and what differences there would be between a Sri Lankan and British hospitals. We had also been told Sri Lanka is a beautiful country with many cultural and historical attractions, which we were all keen to experience for ourselves!

We contacted Dr Tiran Dias, Consultant in Obstetrics and Gynaecology at Ampara General Hospital, through a Professor at St George's under which Dr Dias had worked and gained his MD. He quickly arranged for us to stay and work in Ampara, a town we knew almost nothing about before we got there.

We arrived in Colombo and took the train through the highlands to Ella where we stayed for two nights before driving down to Ampara. Our arrival was slightly delayed by an elephant in the road, a cause of great excitement as we had never seen one before. It was also the start of our medical education in Sri Lanka, as the driver informed us of the dangers of lone elephants and the regularity of elephant attacks. The next morning Dr Dias took us to the hospital and introduced us to the teams we would be placed with for our elective. Having been used to dreary London hospitals and weather walking through Ampara General Hospital was a pleasant surprise. The picturesque grounds with flowers, the deer that lived within the grounds, and the doctors and nurses dressed in such smart uniforms and colourful saris made the grey, cold weather seem a world away. The staff were friendly and welcoming, and we were all excited to start our placement.

Between the four of us we saw a large proportion of the hospital's work. Two of us were with the obstetrics and gynaecology team, while the other two split their time between the preliminary assessment unit, intensive care, general medicine and psychiatry. One of the first things we noticed as being different to the UK was the set up of the general wards. There were many more beds in each ward to accommodate the greater numbers of patients. As many patients travelled a long way to come to hospital some were admitted more for convenience for the patient than clinical need. There also seemed to be a lot more nurses per patient than in the UK which seemed to us key to providing good patient care. The patients themselves often seemed to present later and with more advanced disease than in the UK and often manifested many more clinical signs than we had seen in UK patients. It was quite shocking to us to see what some patients had been putting up with before seeking medical attention. There were also a number of cases which were completely alien to us. We had never heard of a Russels viper before coming to Sri Lanka, yet within a week of being there we had been 
thoroughly educated in the pathology and management of being bitten by such a snake, as well as how to spot one in the wild!

One of the most impressive things we witnessed was the efficiency of the operating theatres. In the UK, delays starting cases are very common. In Ampara, however, the three theatres ran simultaneously with a team of one consultant and four or five juniors who were able to operate on around 15 patients each day. The junior doctors were given far more responsibility than in the UK where they are given much less opportunity to operate independently. Consequently, Sri Lankan doctors seemed to have much more practical experience. The general physicians had a wider knowledgebase and skill-set than doctors in the UK where doctors specialise and subspecialise early in their careers. In Sri Lanka, the general physicians perform echocardiograms, endoscopies and manage dialysis patients whereas in the UK this work is likely to be divided up between many teams. One of the greatest surprises was how many hours the juniors had to work. Some of them seemed to essentially live in the wards for a whole placement, rarely leaving and having very little time off from work. We felt very lucky that 48 hours a week was all we would be legally allowed to work in the UK!

Dr Dias was also keen to get us involved in research projects he was conducting at the hospital. We contributed to a study looking at the awareness of screening for Down's Syndrome amongst patients and staff. This was a multi-centred study involving major hospitals throughout Sri Lanka. It is hoped the findings will be published in a major European journal and will be important in influencing future decisions around screening for Down's Syndrome in Sri Lanka. We also helped collect data on obstetric near misses at the hospital over the last three years in order to look at the outcomes of severe maternal morbidity and improve care for such patients in the future.

However, it was not all ward rounds and research. One of the best things about doing our electives in Ampara was socialising with the hospital staff and we learned more about Sri Lankan customs and culture than we would have done if we had visited the country as simple tourists. We were lucky to be around for the
Vesak celebrations and to see the beautiful lanterns that every department made for the hospital competition. There were also many parties and celebrations within the hospital where we were able to sample delicious traditional Sri Lankan food.

The doctors also helped us explore some of the local attractions, some of which are unknown to tourists and travel guides. We watched the sun set from a nearby temple. We took a boat trip across a local reservoir to a river, watched elephants bathing, swam under waterfalls and had a barbecue in the forest. Another outing was an evening trip to Arugambay where we swam in the sea in the moonlight, and had an excellent meal of crab curry by the beach. These experiences really made the elective special for all of us and we felt lucky to be able to share it with such generous people. As we embark on our careers, we will always remember the time we spent in Sri Lanka. We learnt a lot through our experiences there and saw many things we would not have encountered in the UK.

Overall, it was a wonderful experience, both in and outside the hospital, and would certainly recommend it to other medical students. 